\title{
Construction and expression of a recombinant eukaryotic expression plasmid containing the preS1-preS2-S genes of hepatitis B virus and the granulocyte-macrophage colony stimulating factor gene: A study of its immunomodulatory effects
}

\author{
JUN-YUAN GONG* ${ }^{*}$ XIN LIU*, YAN DONG, TIAN-HONG ZHOU and JUN-WU LI \\ Department of Biotechnology, College of Life Science and Technology, Jinan University, Guangzhou, P.R. China
}

Received August 18,2012; Accepted November 6, 2012

DOI: $10.3892 / \mathrm{br} .2012 .47$

\begin{abstract}
A total of $10-20 \%$ of the population remains unresponsive or weakly responsive to hepatitis B vaccine, which is composed of hepatitis B surface antigen HBsAg (S protein). Therefore, it is necessary to develop a hepatitis B vaccine with a better penetrating and responsive rate. In the present study, a plasmid pVAX1-L-GM was constructed and its immunomodulatory effect of as hepatitis B virus (HBV) DNA vaccine was analyzed through the immunization of BALB/c mice. Immune responses were measured after immunization by anti-HBsAg, proliferation of splenocytes, the number of $\mathrm{CD} 4^{+}$ and $\mathrm{CD}^{+}$molecules, CTL cytotoxicity, cytokines of IFN- $\gamma$ and IL-2 secretion assays. Following the immunization, mice in the pVAX1-L-GM group produced antibody 2 weeks earlier compared to the control plasmid pVAX1 and pVAX1HBsAg groups and antibody levels showed significant differences. Enhanced HBsAg-specific splenocyte proliferation as well as specific cytotoxic activities of splenic CTLs were also detected. Furthermore, pVAX1-L-GM plasmid increased the number of $\mathrm{CD}^{+}$and $\mathrm{CD}^{+}$molecules on the surface of the spleen $\mathrm{T}$ cell and the level of IFN- $\gamma$, IL-2 secretion. pVAX1-L-GM induced a specific immune response in mice and enhanced the immune effect. Thus, a foundation was laid for developing immunogenicity of a better prevention and treatment of HBV via a hepatitis B vaccine.
\end{abstract}

\section{Introduction}

Hepatitis B virus (HBV) infection is one of the most significant public health problems worldwide. Approximately

Correspondence to: Professor Jun-Wu Li, Department of Microbiology and Immunology, Medical College, Jinan University, 601 Huangpu Road, Guangzhou 510632, P.R. China

E-mail: jw188661@163.com

${ }^{*}$ Contributed equally

Key words: hepatitis B virus, preS1-, preS2/S gene, large envelope protein, granulocyte-macrophage colony stimulating factor one-third of the world's 6.0 billion population is estimated to be infected with HBV (1), including 350-400 million chronic carriers of this virus (2). The HBV vaccine applied at present is primarily composed of HBsAg (S protein) expressed by Chinese hamster ovary $(\mathrm{CHO})$ cells and beer yeast. A total of $10-20 \%$ of the population is known to be unresponsive or weakly responsive to the HBV vaccine or even not to produce antibodies at all $(3,4)$. Vaccination is crucial in the prevention of HBV infection and there are no specific therapies to manage it. Therefore, it is crucial to develop a hepatitis B vaccine with a better penetrating and responsive rate.

Hepatitis B vaccine made by recombinant DNA techniques has the same degree of safety as the recombinant subunit vaccine and the same efficacy to induce immune response as live attenuated vaccine. It is relatively simple to clone and is a purified DNA with no need for a synthetic protein in vitro, and can sustain long-term immune efficacy (5). The recombinant DNA vaccine is also relatively cost-effective and convenient to transport and preserve, thus it is a promising approach for the vaccine development. HBV DNA vaccination may induce the $\mathrm{CD}^{+} \mathrm{T}$ cell as well as dominant Th1 phenotype among the splenic lymphocytes, to elicit strong CTL and protective antibody levels (6).

To overcome traditional HBsAg vaccine immunogenicity defects, HBV large envelope protein (L protein) was selected as a dominant antigen, while granulocyte-macrophage colony stimulating factor (GM-CSF) acted as an immune adjuvant to enforce antibody response and construct an eukaryotic expression plasmid pVAX1-L-GM containing preS1, preS2 and $S$ genes of the L protein and immune adjuvant GM-CSF. After the successful expression of the vaccine into the L-02 cell line, immune responses were stimulated in mice to lay a foundation for the development of a novel type of hepatitis B DNA vaccine.

\section{Materials and methods}

Ethics. The present study was conducted in the Department of Microbiology and Immunology of the Medical College of the Jinan University (Guangzhou, China). The Ethics Committee of The First Affiliated Hospital of the Jinan University (Guangzhou, China) approved the animal procedures and the experimental protocol. 
Construction and identification of recombinant plasmid. Based on the CDS sequence of the preS1-preS2-S gene (GI:157091234), designed the primers 5'-CAGCTAGCATGG GAGGTTGGTCTTCCAAA-3' upstream) (NheI) (Takara Bio Inc., Otsu, Japan) and 5'-GGCGGAAGCTTAATGTATACCC AAAGAC-3' (downstream) (HindIII) with appropriate restriction endonuclease sites. Hepatitis B DNA extraction was obtained from the hepatitis B surface antigen-positive serum (The First Affiliated Hospital of the Jinan University) using phenol/chloroform extraction methods and used as a template to amplify HBV preS1 preS2 S region. The coding sequences of these GM-CSF fragments were synthesized using PCR from pORF-GM-CSF using specific primers, upstream: 5'-CCA AGCTTGGTGGCGGTGGAAGCGGCGGTGGCGGAAGC GGCGGTGGCGGCAGCTGGCTGCAGAGCCTGCT-3' (HindIII and Linker), downstream: 5'-CGGAATTCTCACTCC TGGACTGGCTC-3' (EcoRI), and cloned into pVAX1 using the standard cloning techniques. PCR and restriction endonuclease assay were used to screen and identify positive clones. DNA sequencing analysis (Sangon Biological Engineering and Technology and Service Company, Shanghai, China) of the recombinant pVAX1-L-GM identified the sucessful constructions of recombinant plasmid pVAX1-L-GM.

Cell transfection. L-02 cells were digested with $0.25 \%$ trypsin and diluted to $2 \times 10^{5}$ cells $/ \mathrm{ml}$ and plated to 6-well plates with $2 \mathrm{ml}$ medium per well. Then, when cells were $70-80 \%$ confluent, $4 \mu \mathrm{g}$ purified plasmid were transfected into the prepared cells using $8 \mu$ l Lipofectamine 2000 reagent (Invitrogen, Carlsbad, CA, USA).

Immunocytochemical staining. Non-transfected cells were considered as the blank, while pVAX1-transfected cells as the negative comparison. Immunocytochemical staining was performed according to the manufacturer's instructions (Boster Biological Technology, Ltd., Wuhan, China), and mouse anti-HBsAg antibody was used as the primary antibody.

Western blot analysis. Western blot analysis of fusion proteins was performed according to the standard procedure. The purified protein was separated on $12 \%$ SDS-PAGE and transferred to nitrocellulose membrane. Anti-HBsAg mAb antibody (Beijing Biosynthesis Biotechnology Co., Beijing, China) at a dilution of 1:1000 or Anti-GM-CSF mAb was used as the primary antibody to detect the presence of protein. Blots were developed using the ECL (Thermo Fisher Scientific, Inc., Rockford, IL, USA) method with HRP-labeled rabbit anti-mouse $\operatorname{IgG}$ at a dilution of 1:5000.

ELISA assay protein levels of GM-CSF. Double-antibody sandwich ELISA (DAS-ELISA) was used to detect the GM-CSF protein level according to the manufacturer's instructions (R\&D Inc., Minneapolis, MN, USA). The results were presented as the mean \pm standard deviation (SD), and statistically significant differences between values were analyzed using the SPSS 13.0 software. $\mathrm{P}<0.05$ was considered to indicate a statistically significant difference.

Animal immunization. Female BALB/c mice ( $\mathrm{n}=30 ; 6-8$ weeks old) were purchased from the Experimental Animal Center of the Jinan University (Guangzhou, China) and divided equally into 3 groups ( $\mathrm{n}=10 /$ group). Mice were immunized intramuscularly individually with pVAX1-L-GM, pVAX1 and pVAX1HBsAg. The mice were then injected with a dose of $100 \mu \mathrm{g} / 100 \mu \mathrm{l}$ plasmid pVAX1-L-GM, pVAX1 and pVAX1HBsAg, 3 times every second week. Blood was obtained from the tail each week after immunization. The spleens of each mouse in the vaccinated groups were removed aseptically at week 13 after the first immunization.

Detection of specific anti-HBSAb antibodies using the ELISA test. After the first-immunization, serum was collected every week using the tail vein bleeding method. Absorbance at $450 \mathrm{~nm}$ was measured in a microplate reader, according to the manufacturer's instruction of the Mouse HBsAb ELISA kit (Wuhan EIAab Science Co., Ltd., Wuhan, China). Levels of serum antibody in immunized mice were monitored for 12 weeks.

Proliferation of splenocytes. Lymphocyte proliferation of immunized mice was measured by MTT assay [3-(4,5-dimethylthiazol-2-yl)-2,5-diphenyl tetrazolium bromide]. At week 13 following the first immunization, mice were sacrificed, their spleens were aseptically removed and splenocytes were prepared as single-cell suspensions. The cells were cultured in triplicate using 96-well round-bottom plates at $2 \times 10^{7}$ cells per well. RPMI-1640 (Gibco, Paisley, UK) supplemented with $10 \%$ fetal calf serum (FCS) was added to each well, and stimulated with HBsAg at a final concentration of $10 \mu \mathrm{g} / \mathrm{ml}$. Lymphocytes stimulated with the medium alone were used as the negative control. The cells were incubated for $48 \mathrm{~h}$ at $37^{\circ} \mathrm{C}$ in a humid atmosphere of $5 \% \mathrm{CO}_{2}$, then $20 \mathrm{ml}$ of MTT $(5 \mathrm{mg} /$ $\mathrm{ml}$ ) (Sigma-Aldrich, Shanghai, China) was added to each well. Following additional incubation for $4 \mathrm{~h}$, the supernatant was carefully aspirated and $200 \mu \mathrm{l}$ of dimethylsulfoxide (DMSO) was added into each well and absorbance of the soluble formazan was measured at $570 \mathrm{~nm}$ using an automatic microplate reader (Bio-Rad, Hercules, CA, USA).

Cytotoxicity assay. Splenocytes obtained from mice at week 13 , following the first immunization were cultured in 24-well plates with complete culture RPMI-1640 medium [with $10 \%$ FBS, $50 \mu \mathrm{M}$ 2-mercaptoethanol, $10 \mathrm{mM}$ HEPES, $2 \mathrm{mM}$ L-glutamine, 100 units of penicillin per $\mathrm{ml}$ and $100 \mu \mathrm{g}$ of streptomycin per ml]. Complete culture RPMI-1640 medium was used containing $5 \mu \mathrm{g} / \mathrm{ml}$ Concanavalin A (Dingguo Biotech, Beijing, China) and $10 \mathrm{U} / \mathrm{ml}$ of IL-2 (Pepro Tech, London, $\mathrm{UK}$ ) to culture splenocytes in vitro for 2 days as the effector. The stimulator cells, harvested from naive mice, were pulsed with the final concentration of $20 \mu \mathrm{g} / \mathrm{ml}$ of $\mathrm{HBV}$-specific peptide for $4 \mathrm{~h}$ at $37^{\circ} \mathrm{C}$ in $5 \% \mathrm{CO}_{2}$, and were then treated with $80 \mu \mathrm{g} / \mathrm{ml}$ mitomycin $\mathrm{C}$ for another $2 \mathrm{~h}$. The cells were washed extensively with RPMI-1640 medium. The effector cells $\left(4 \times 10^{7}\right.$ cells) were incubated with stimulator cells at an effector-stimulator ratio of 10:1 for 7 days in culture medium containing $10 \mathrm{U} / \mathrm{ml}$ recombinant IL-2 (Peprotech, Rocky Hill, NJ, USA) at $37^{\circ} \mathrm{C}$ in $5 \% \mathrm{CO}_{2}$. The target cells were prepared by P815 cells (mouse mastocytoma cell line, Shanghai Institute of Biochemistry and Cell Biology of the Chinese Academy of Sciences) pulsed with $\mathrm{HBV}$-specific peptide for $4 \mathrm{~h}$ at $37^{\circ} \mathrm{C}$ in 
$5 \% \mathrm{CO}_{2}$. The cytotoxic activity was tested by non-radioactive $\mathrm{LDH}$ release assay. The assays were performed in triplicate with $1 \times 10^{4}$ target cells/well incubated with effector cells at various effector cell/target cell (E:T) ratios of 100:1, 50:1, 25:1 and 12.5:1 in 96-well round-bottom plates, according to the Non-Radioactive Cytotoxicity Assay Kit (Promega, Madison, WI, USA). The absorbance values from the supernatants were recorded at $490 \mathrm{~nm}$ using an ELISA microplate reader.

Analysis of the molecules of $\mathrm{CD}^{+}$and $C D 8^{+}$on the surface of $T$ cell. At week 13 following immunization, the mice were sacrificed and their spleens were removed aseptically. Phosphate-buffered saline (PBS) buffer $(0.1 \mathrm{mmol} / \mathrm{l})$ was used to wash the spleen cells and cell suspension was collected. The $\mathrm{CD}^{+} / \mathrm{CD}^{+}$detection kit (Beckman Coulter, Inc., Brea, CA, USA) required the volume of $100 \mu \mathrm{l}$ of each sample intake, in order to detect the number of $\mathrm{CD}^{+}, \mathrm{CD}^{+}$molecules on the surface of spleen $\mathrm{T}$ cells using the Epics XL flow cytometry (Beckman Coulter, Miami, FL, USA).

Cytokines of IFN- $\gamma$ and IL-2 secretion assays. The splenocytes of immunized mice were cultured following the same procedure in the proliferation assays for $72 \mathrm{~h}$. Following incubation, the supernatant from each well was removed for evaluation of secreted IFN- $\gamma$ and IL-2 levels using ELISA. The concentrations of IFN- $\gamma$ and IL-2 in the culture supernatant were measured using murine cytokine ELISA kits (R\&D Systems, Minneapolis, MN, USA). The limit of the detection was $2 \mathrm{pg} / \mathrm{ml}$.

Statistical analysis of data. Measurement data show the mean \pm SD. The statistical software SPSS was used to perform statistical analysis. Differences between groups were analyzed using stochastic analysis of variance. $\mathrm{P}<0.05$ was considered to indicate a statistically significant difference.

\section{Results}

In vitro expression of the recombinant plasmid. To determine whether or not the recombinant plasmid pVAX1-L-GM was expressed in vitro, L-02 cells were transiently transfected with pVAX1-L-GM or pVAX1 and their expression at a protein level was detected using western blot analysis and ELISA assay, respectively. Western blot analysis indicated a protein level of $\sim 64 \mathrm{kDa}$, as expected (Figs. 1 and 2). Expression values of GM-CSF proteins in the $\mathrm{pVAX} 1$ control group were $1.382 \pm 0.081 \mathrm{pg} / \mathrm{ml}$ and $1.382 \pm 0.081 \mathrm{pg} / \mathrm{ml}($ mean $\pm \mathrm{SD}, \mathrm{n}=3$ ). While the expression values of the GM-CSF proteins of the pVAX1-L-GM-transfected group were $153.073 \pm 4.20$ and $193.124 \pm 3.943 \mathrm{pg} / \mathrm{ml}$, compared to the negative control groups (cell control and pVAX1), the results were considered statistically significant $(\mathrm{P}<0.05)$ (Fig. 3).

Detection of specific anti-HBsAb antibodies using ELISA. To assess the effect of the pVAX1-L-GM DNA vaccine on the humoral responses in mice, blood samples were collected using the tail vein bleeding method each week following the first immunization, and the sera were isolated. The presence of anti-HBsAg-specific antibodies in sera was analyzed by ELISA. Specific antibody response was detectable in the

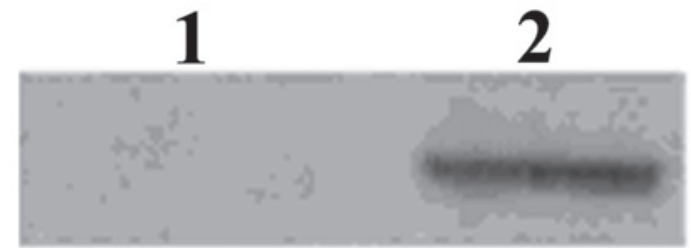

Figure 1. Western blot analysis of expressed L-GM protein in L-02 cells with anti-HBsAg. Lane 1, lysate supernatant from pVAX1-transfected cells incubated by anti-HBsAg mAb; lane 2, lysate supernatant from pVAX1-L-GMtransfected cells incubated with anti-HBsAg mAb.

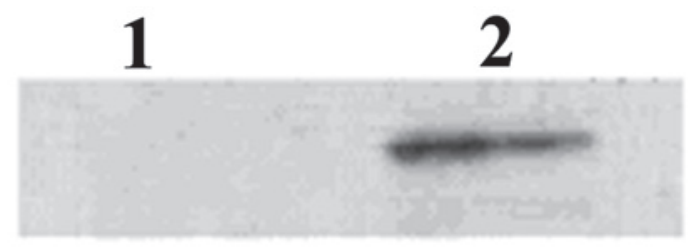

Figure 2. Western blot analysis of expressed L-GM protein in L-02 cells with anti-GM-CSF. Lane 1, lysate supernatant from pVAX1-transfected cells incubated by anti-GM-CSF; lane 2, lysate supernatant from pVAX1-L-GMtransfected cells incubated with anti-GM-CSF mAb.

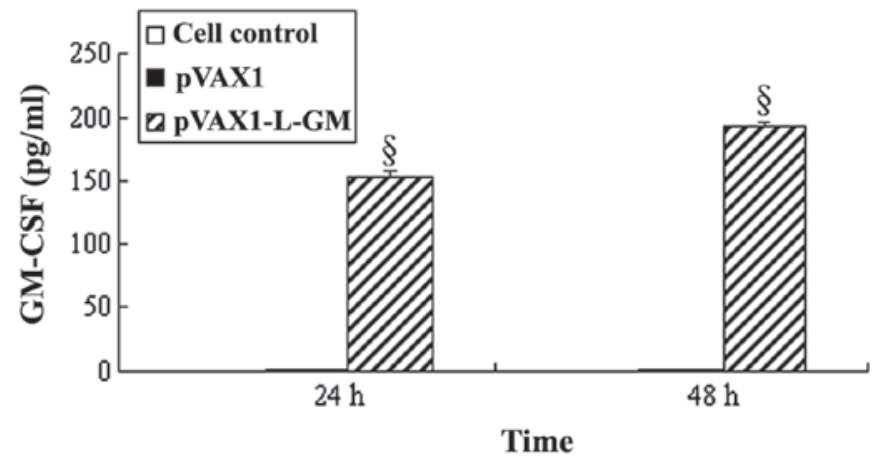

Figure 3. ELISA analysis of GM-CSF protein in L-02 cells. Transfected cells culture supernatants were collected separately at 24 and $48 \mathrm{~h}$, using granulocyte-macrophage colony stimulating factor (GM-CSF) protein ELISA examination reagent box, according to the manufacturer's instruction. pVAX1-L-GM-transfected cells were able to express and secrete GM-CSF proteins. ${ }^{\S} \mathrm{P}<0.05$ compared to the negative control groups (cell control, pVAX1).

pVAX1-L-GM and pVAX1HBsAg groups (Fig. 4). Statistical analysis of antibody levels of pVAX1-L-GM and pVAX$1 \mathrm{HBsAg}$ groups was performed and an enhanced antibody response was observed in the pVAX1-L-GM group, and the difference was statistically significant $(\mathrm{P}<0.05)$. The pVAX1-L-GM group produced antibody 2 weeks earlier than the control plasmid pVAX1 and pVAX1HBsAg groups.

Proliferation of splenocytes. To determine whether or not the pVAX1-L-GM DNA vaccine influenced cell-mediated immunity, a single-cell suspension of lymphocytes was prepared from immunized mice at week 13 after immunization. As shown in Fig. 5, mice immunized with pVAX1-L-GM elicited the highest level of splenocyte T-cell proliferation compared to the pVAX1HBsAg and pVAX1 groups $(\mathrm{P}<0.05)$. 


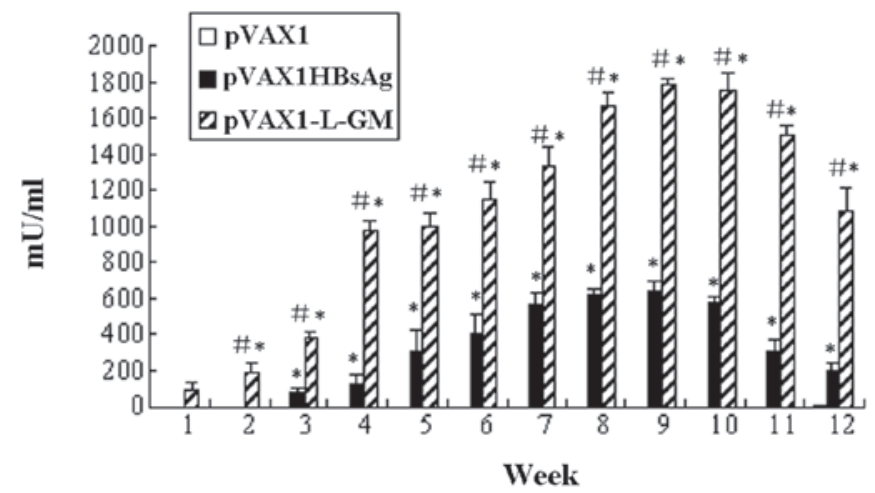

Figure 4. Level of specific anti-HBsAb antibodies in mice. After immunizing mice with pVAX1-L-GM, pVAX1 and pVAX1HBsAg, serum was collected each week by orbital bleeding. The levels of serum antibody were monitored using the $\mathrm{HBsAb}$ detection kit, according to the manufacturer's instructions. Results are shown as the mean \pm standard deviation. The level of antibody of the pVAX1-L-GM group was significantly $(\mathrm{P}<0.05)$ higher compared to other groups. ${ }^{\text {}} \mathrm{P}<0.05$ compared to the pVAX1HBsAg group. " $\mathrm{P}<0.05$ compared to the pVAX1 control group.

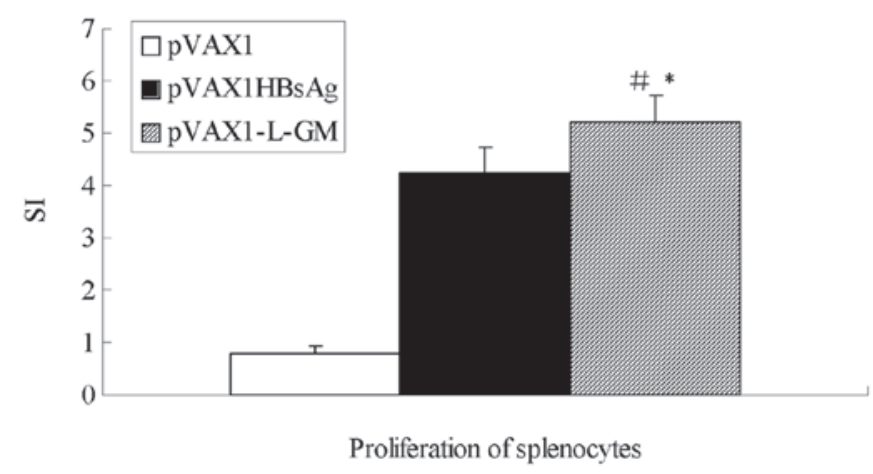

Figure 5. Lymphoproliferative response to the three types of vaccines. Lymphocytes isolated from the spleens of the mice immunized with pVAX1-L-GM, pVAX1 and pVAX1HBsAg were cultured in 96-well flat bottom plates. The cells were stimulated with HBsAg in vitro. Lymphocytes stimulated with the medium alone were used as the negative control. The proliferative response was measured by MTT assay. The stimulation index (SI) value was calculated as the ratio of absorbent value at $570 \mathrm{~nm}$ of pools incubated with $\mathrm{HBsAg}$ to that of pools incubated with medium only. Data are presented as the mean \pm standard deviation. ${ }^{*} \mathrm{P}<0.05$ compared to a pVAX1 HBsAg group. ${ }^{*} \mathrm{P}<0.05$ compared to the pVAX1 control group.

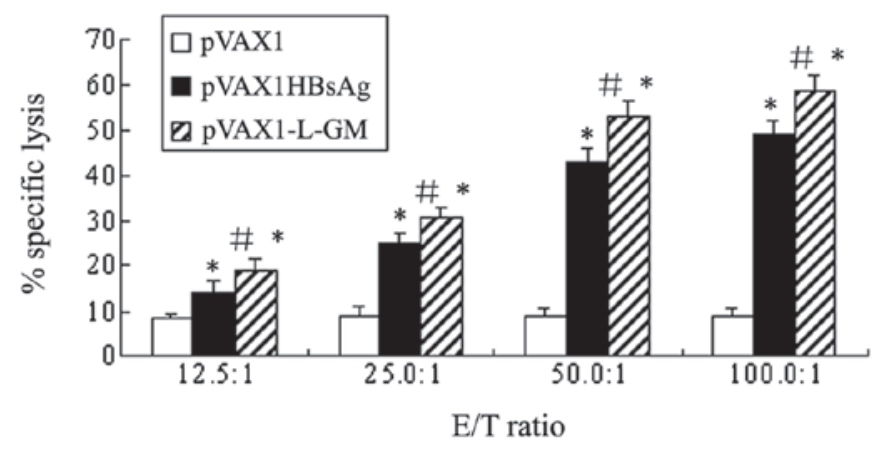

Figure 6. HBsAg-specific CTL response. BALB/c mice were injected with pVAX1-L-GM, pVAX1 and pVAX1HBsAg. Splenocytes from the mice in each group were harvested and CTL responses were measured using autologous P815 cells incubated with HBsAg-specific peptide as the target cells. Effector cell-target cell ratios are indicated on the abscissa. The percentage of specific lysis is reported on the vertical axis. ${ }^{~} \mathrm{P}<0.05$ compared to the pVAX1HBsAg group. ${ }^{*} \mathrm{P}<0.05$ compared to the pVAX1 control group.

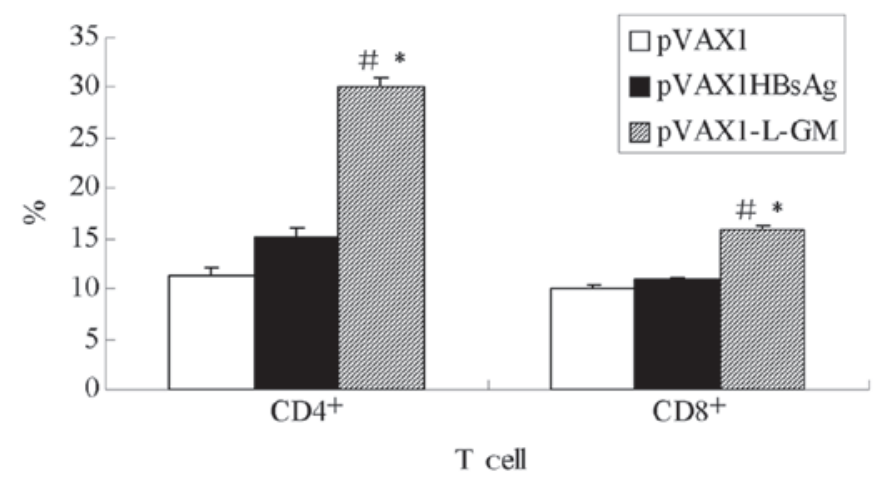

Figure 7. Molecules of $\mathrm{CD}^{+}$and $\mathrm{CD}^{+}$on the surface of $\mathrm{T}$ cells. After splenocytes were produced following pre-test treatments, $\mathrm{CD} 4^{+} / \mathrm{CD}^{+}$were detected by flow cytometry using a detection kit according to the manufacturer's instruction, adjusting the cell concentration to $1 \times 10^{7} / \mathrm{ml}$, taking $100 \mu \mathrm{l}$ of each reagent. Results are presented as the mean \pm standard deviation. ${ }^{\#} \mathrm{P}<0.05$ compared to the pVAX1HBsAg group. ${ }^{*} \mathrm{P}<0.05$ compared to the pVAX1 control group.

Cytotoxicity assay. In the protection against or eradication of viruses or other intracellular pathogens, specific cytotoxic responses have been previously demonstrated to be a key factor. To analyze the ability of recombinant pVAX1-L-GM to enhance the HBsAg-specific CTL response, splenic cells, derived from the immunized mice 13 weeks after immunization, were restimulated specifically by naive mice splenocytes pulsed with HBsAg-specific peptides in vitro for 7 days. P815 cells pulsed with HBsAg-specific peptides were used as target cells. The cytotoxic activity was tested using non-radioactive $\mathrm{LDH}$ release assay and the specific lysis rates are shown in Fig. 6. HBsAg-specific CTL was detectable in the mice immunized with the HBV DNA vaccine pVAX1-L-GM plasmids compared to the pVAX1HBsAg or pVAX1 groups at the E/T ratio of 100:1 $(\mathrm{P}<0.05)$. The specific CTL activities increased significantly in the pVAX1-L-GM and pVAX1HBsAg groups compared to the pVAX1 group, while the strongest CTL response was detected at the $\mathrm{E} / \mathrm{T}$ ratio of 100:1 $(\mathrm{P}<0.01)$. The results demonstrated that cellular immunity was markedly enhanced by pVAX1-L-GM DNA and pVAX1HBsAg DNA vaccine plasmids.

Analysis of the molecules of $\mathrm{CD}^{+}, \mathrm{CD}^{+}$on the surface of $T$ cell. To evaluate the subsets of $\mathrm{T}$ cells, total $\mathrm{T}$ cells were isolated at week 13 following immunization and re-stimulated in culture with HBsAg. These cells were then analyzed using FACS with a gate set on $\mathrm{CD}^{+}$and $\mathrm{CD} 8^{+} \mathrm{T}$ cells. As shown in Fig. 7, the number of $\mathrm{CD}^{+}$and $\mathrm{CD}^{+}$molecules on the surface of spleen $\mathrm{T}$ cell produced by the pVAX1-L-GM group was higher compared to the pVAX1HBsAg group, and the difference was statistically significant $(\mathrm{P}<0.05)$, indicating that the $G M-C S F$ gene enhanced cell immune function.

Cytokines of IFN- $\gamma$ and IL-2 secretion assays. We quantified the production of the cytokines IFN- $\gamma$ and IL-2 released from splenocytes from immunized mice re-stimulated with $\mathrm{HBsAg}$ in vitro. Mice immunized with DNA vaccine pVAX1-L-GM elicited a significant enhancement of IFN- $\gamma$ and IL-2 production, even significantly higher compared to the pVAX1HBsAg 

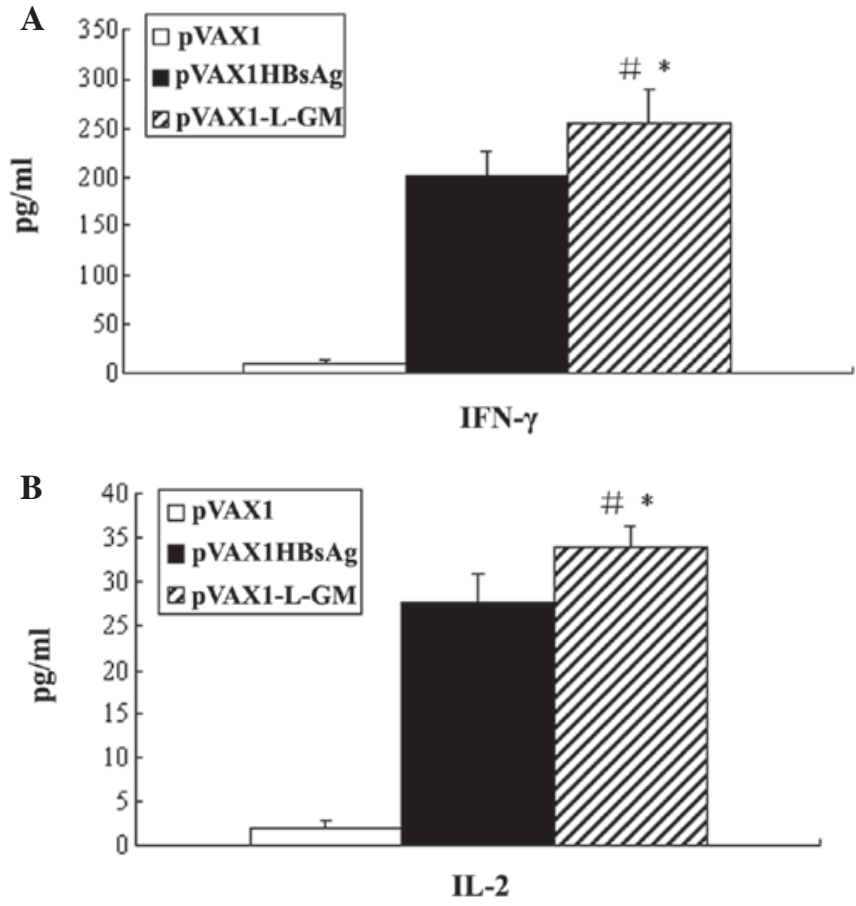

Figure 8. ELISA analysis of IFN- $\gamma$ and IL-2 concentration of the lymphocyte secreted. The splenocytes collected from the mice immunized with pVAX1-L-GM, pVAX1 and pVAX1HBsAg were seeded in 96-well flatbottom plates and re-stimulated with $\mathrm{HBsAg}$ in vitro, and the plate was incubated at $37^{\circ} \mathrm{C}$ for $60 \mathrm{~h}$ with $5 \% \mathrm{CO}_{2}$, IFN- $\gamma$ and the IL-2 concentration of the lymphocyte secreted into the medium was measured using an ELISA kit, according to the manufacturer's instructions. ${ }^{~} \mathrm{P}<0.05$ compared to the pVAX1HBsAg group. " $\mathrm{P}<0.05$ compared to the pVAX1 control group.

group $(\mathrm{P}<0.05)$ (Fig. 8). This finding suggested that this DNA vaccine resulted in stronger Th1-type cell immune response.

\section{Discussion}

In this study, the $\mathrm{L}$ protein was used as an antigen. Compared to the gene of the $\mathrm{S}$ protein, the gene of $\mathrm{L}$ protein has more preS1 and preS2 gene. Previous studies demonstrated that the preS1 (21-47aa) is the liver cell receptor binding site, which binds the HBV into the host cell membrane. Previous studies have also demonstrated that the preS1 peptide has several $\mathrm{T}$ - and $\mathrm{B}$-cell epitopes, and that anti-preS1 serum is able to neutralize the toxicity of $\mathrm{HBV}$ and protect against HBV infection gorillas (7-9). PreS2 (120-145aa) sequence also contains multiple epitopes which are able to neutralize the virus. It is able to mediate HBV adhesion and the invasion of liver cells, and with strong immunogenicity, is able to induce the generation of neutralizing antibodies and protective immunity. Containing the specific $\mathrm{T}$ and $\mathrm{B}$ lymphocytes binding site, it is able to break the body immune tolerance of existing HBsAg vaccine (10-12).

There has been an increasing demand for gene adjuvant therapy both in the treatment and prevention of HBV in recent years, thus $G M-C S F$ as a preponderant adjuvant is important in activating endotheliocytes and macrophagocytes through various mechanisms. It can also regulate the amount and function of antigen-presenting cells and enhance the cytoactivity of CTL and NK, thereby strengthening the immune level of the hepatitis B vaccine. Several clinical trials showed that $G M-C S F$ is able to increase antibody levels in subjects in a rapid, effective and constant manner (13-15). Therefore, $G M-C S F$ was used as an immune adjuvant, for studies of gene adjuvant in the field of DNA vaccine immunopotency improvement.

In this study, we used vector-pVAX1, which was approved by the Food and Drug Administration (FDA) and could be used in the late stages of human disease, to clone a recombinant plasmid which containing the gene of the $\mathrm{L}$ protein and the GM-CSF gene, through Linker to connect the fusion gene consisting of glycine $(\mathrm{G})$ and serine $(\mathrm{S})$ to five peptides (16). Thus, the recombinant plasmid was transfected into L-02 cells using liposome transfection methods, confirming the successful construction of pVAX1-L-GM by restriction enzyme digestion and sequencing, and also confirming the stable expression of fusion protein using western blot analysis and ELISA.

To determine whether or not the pVAX1-L-GM plasmid has any effect on the cell and humoral immune responses in $\mathrm{BALB} / \mathrm{c}$ mice, three groups of mice were immunized in the study with negative control plasmid (pVAX1 group), positive control plasmid (pVAX1HBsAg group) and recombinant plasmid (pVAX1-L-GM group). The level of antibody was then detected and the results showed that the antibody level of the experimental pVAX1-L-GM group was higher compared to the empty vector pVAX1 and positive control pVAX1 HBsAg groups. The difference was statistically significant $(\mathrm{P}<0.05)$. Additionally, studies have demonstrated that the presence of antibodies in the pVAX1-L-GM group was recorded two weeks earlier compared to the pVAX1HBsAg group. Splenocytes from mice in the three groups were evaluated for antigen-specific proliferation as well as the CTL component of immune response by the specific killing of syngeneic target cells pulsed with a recognized CTL epitope peptide. The number of $\mathrm{CD}^{+}$and $\mathrm{CD} 8^{+}$molecules on the surface of spleen T cells and the level of IFN- $\gamma$ and IL-2 was also detected. The strongest HBsAg-specific proliferative activity in the pVAX1-L-GM group was in concordance with the highest level of CTL activity and $\mathrm{CD} 4^{+}, \mathrm{CD} 8^{+}$molecules and IFN- $\gamma$, IL-2 production in the two groups. The level of CD8 ${ }^{+}$ on cytotoxic $\mathrm{T}$ lymphocyte was increased, indicating that cell immunity was significantly enhanced after immunization by the fusion $L-G M$ gene. The results indicated that the plasmid pVAX1-L-GM is able to enhance the specific cell and humoral immune responses in mice.

Possible reasons for these results are that the $\mathrm{L}$ protein has several advantageous features of its antigens, compared to the commercially available hepatitis B HBsAg vaccine whose component contains only S antigen. GM-CSF as an adjuvant is able to stimulate the activation of antigen-presenting cells, promote the secretion of IL-2 and the proliferation of $\mathrm{CD} 4^{+}$ and $\mathrm{CD}^{+}$cells, thus it is likely to be important in enhancing the DNA vaccine induction of humoral and cell immune responses.

In summary, the results suggest that $\mathrm{L}$ protein stimulated the cell and humoral immune response following immunization of mice with $L-G M$ fusion gene. The immune effect of vaccine pVAX1-L-GM was superior to that of pVAX1HBsAg vaccine since cell immunity is considered to be the most essential host immune response to eradicating the virus. 
Therefore, this study provided a novel method to enhance the effect of hepatitis B DNA vaccine, as well as an effective means to develop hepatitis B DNA vaccine as a prevention and treatment vaccine. Additional investigations into the immune protection of the vaccine may have better prospects.

\section{Acknowledgements}

The present study was supported by the Research Fund of the Science and Technology Plan of Guangdong Province (no. A20101006-2006) and the Major National Science and Technology (S\&T) Special Projects (no. 2008ZX10002-009).

\section{References}

1. Poorolajal J, Mahmoodi M, Majdzadeh R, Nasseri-Moghaddam S, Haghdoost A and Fotouhi A: Long-term protection provided by hepatitis $\mathrm{B}$ vaccine and need for booster dose: a meta-analysis. Vaccine 28: 623-631, 2010.

2. Shen T, Yan XM,Zou YL, Gao JM and Dong H: Virologic characteristics of hepatitis B virus in patients infected via maternal-fetal transmission. World J Gastroenterol 14: 5674-5682, 2008.

3. Kubba AK, Taylor P, Graneek B and Strobel S: Non-responders to hepatitis B vaccination: a review. Commun Dis Public Health 6: 106-112, 2003.

4. Jin L, Wang G, Zhao X, et al: Characterization and immune effect of the hepatitis B-BCG combined vaccine for using a needle innoculation. Vaccine 28: 6041-6051, 2010.

5. Hanif SN, Al-Attiyah R and Mustafa AS: DNA vaccine constructs expressing Mycobacterium tuberculosis-specific genes induce immune responses. Scand J Immunol 72: 408-415, 2010.

6. Qing Y, Chen M, Zhao J, et al: Construction of an HBV DNA vaccine by fusion of the GM-CSF gene to the HBV-S gene and examination of its immune effects in normal and HBV-transgenic mice. Vaccine 28: 4301-4307, 2010.
7. Park JH, Cho EW, Lee YJ, Shin SY and Kim KL: Determination of the protective effects of neutralizing anti-hepatitis B virus (HBV) immunoglobulins by epitope mapping with recombinant HBV surface-antigen proteins. Microbiol Immunol 44: 703-710, 2000.

8. Lian M, Zhou X, Chen B, Li C, Gu X, Luo M and Zheng X: Identification of the critical regions in hepatitis $\mathrm{B}$ virus preS required for its stability. J Pept Sci 14: 307-312, 2008.

9. Petersen J, Dandri M, Mier W, et al: Prevention of hepatitis B virus infection in vivo by entry inhibitors derived from the large envelope protein. Nat Biotechnol 26: 335-341, 2008.

10. Park JH, Lee MK, Kim HS, Kim KL and Cho EW: Targeted destruction of the polymerized human serum albumin binding site within the preS2 region of the HBV surface antigen while retaining full immunogenicity for this epitope. J Viral Hepat 10: 70-79, 2003.

11. Yin Y, Lin F, Zhuang Q, Liu L and Qian C: Generation of full-length functional antibody against preS2 of hepatitis B virus in hepatic cells in vitro from bicistrons mediated by gutless adenovirus. Bio Drugs 23: 391-397, 2009.

12. Salyaev RK, Stolbikov AS, Rekoslavskaya NI, Shchelkunov SN, Pozdnyakov SG, Chepinoga AV and Hammond RV: Obtaining tomato plants transgenic for the preS2-S-HDEL gene, which synthesize the major hepatitis B surface antigen. Dokl Biochem Biophys 433: 187-190, 2010.

13. Cruciani M, Mengoli C, Serpelloni G, Mazzi R, Bosco O and Malena M: Granulocyte macrophage colony-stimulating factor as an adjuvant for hepatitis B vaccination: a meta-analysis. Vaccine 25: 709-718, 2007.

14. Rizza P, Ferrantini M, Capone I and Belardelli F: Cytokines as natural adjuvants for vaccines: where are we now? Trends Immunol 23: 381-383, 2002.

15. Sasaki MG, Foccacia R and de Messias-Reason IJ: Efficacy of granulocyte-macrophage colony-stimulating factor (GM-CSF) as a vaccine adjuvant for hepatitis B virus in patients with HIV infection. Vaccine 21: 4545-4549, 2003.

16. Gustavsson M, Lehtiö J, Denman S, Teeri TT, Hult K and Martinelle M: Stable linker peptides for a cellulose-binding domain-lipase fusion protein expressed in Pichia pastoris. Protein Eng 14: 711-715, 2001. 